\title{
Temporal and Spatial Changes in Astrocytes in Different Brain Regions Following Intrauterine Ischemia in Neonatal Wistar Rats
}

\author{
Xiaoming Lv, Dan Dang, Zhiyan Xu, Siwen Li and Hui $\mathrm{Wu}^{*}$ \\ Department of Neonatology, the First Hospital of Jilin University, Changchun 130021, China \\ ${ }^{*}$ Corresponding author
}

\begin{abstract}
Early stage of different degrees of HI can promote the proliferation of astrocytes and developed the neuroprotective effect. But overactive astrocytes will lead to glutamic acid, nitric oxide, oxygen radicals increasing continually and the release of abundant harmful factors, which not only brought about the death of themselves, meanwhile also had adverse effect on other neural cells.
\end{abstract} rats

Keywords - astrocytes; intrauterin e ischemia; neonatal wistar

\section{INTRODUCTION}

Perinatal hypoxia-ischemia brain damage (HIBD) is a serious threat to the newborn health and partial survivors left cerebral palsy, epilepsy, mental retardation and other central nervous system sequelae. Previously, the study of HIBD mostly concentrated on neurons. However, recently it is reported that astrocytes (AST) play a key role in the process of hypoxia-ischemia (HI) whose changes are the earliest and the most significant in the central nervous system. Newborn rat brain has stronger plasticity than adult rat brain and is more sensitive to HI. In the past, the study related to changes of AST after HI was focused on adult rats and few studies used newborn rats, even though neonatal HI studies usually adopted seven-day-old model, which is equal to the human full-term infants. Our study is aimed to elaborate the changes of AST in rats born within 28 days after being exposed varying degrees of intrauterine HI. By observing the number of astrocytes labeled by glial fibrillary acidic protein (GFAP) to explore the spatial and temporal patterns in the perinatal acute mild and severe intrauterine HI.

\section{MATERIALS AND METHODS}

This study was performed in the Department of Pathology, Bethune Medical College of Jilin University, Changchun, China between February 2009 and January 2010. Ethical approval was received prior to conducting this study. Ninety-day-old female Wistar rats, weighing $280 \pm 20 \mathrm{~g}$, were provided by Gaoxin Experimental Research Center of Medical Animal, Changchun, China. A rat model of varying degrees of acute intrauterine distress was successfully constructed by ligating the bilateral uterine arteries of pregnant rats previously [1]. According to the duration of ischemia, 60 pregnant rats were divided into 3 groups: control group, whose uterus was surgically exposed, but whose uterine arteries were not occluded; mild HI group, and severe HI group, whose vessels of bilateral uterine horns and uterine body were completely clamped for 5 and 25 minutes. The newborn rats were breastfed by other female rats who delivered normally the day before. Ten newborn rats from each group (control, mild HI, and severe $\mathrm{HI}$ ) were selected randomly at $0.5,3,6$, and 24 hours, 3, 5, 7, 9, 11, 14, 21, and 28 days following brain ischemia-reperfusion, and were sacrificed using cervical dislocation. The brain tissues were extracted and used for immunohistochemistry as previously described [2]. Astrocytes were labeled using rabbit anti-GFAP immunohistochemistry (Fuzhou Maxim Biotech Inc., Fuzhou, China.). Under a 20X object lens, 6 non-overlapping visual fields were selected for each slice. Cells with brown cell membranes were regarded as positive cells. The positive cells were scanned with a MICROPHOT-FXA camera (Nikon, Tokyo, Japan), and stored in an image analysis system. Astrocyte abundance was expressed according to the average number of positively stained cells within the reference area of each image obtained from 6 visual fields of each section. All data are expressed as mean \pm standard deviation (SD). Statistical analyses were performed using the SAS 6.12 statistical software package (SAS Inc., Cary, NC, USA), and comparisons between groups were conducted using ANOVA tests and the LSD test, a significant difference was assumed at $\mathrm{p}<0.05$.

\section{RESULTS}

In the control group (Figures1A-1c), GFAP positive cells were undetectable at $0.5,3$ hours, and were slightly expressed at 6 hours postnatally. The positive cells were sparse and slightly stained with small cell body and few or no protrusions. There is no significant changes at 6 hours $\sim 3$ days; but the number of GFAP positive cells start increasing at postnatal 7 days, accompanied with the cell body enlarging, the protrusions increasing, elongating, thickening and the staining slightly deepened. The staining of GFAP positive cells were significantly deepened and protrusions elongated at 14 days in the region of hippocampal, while the changes in cortex and striatum were not significant. The expression of GFAP positive cells in hippocampus was stronger than the cortex. The positive cells in the dentate gyrus were regularly distributed from inter to outer layers, whose protrusions were perpendicular to the surface and parallel to each other, mostly are single protrusion and less protrusions. The positive cells in other regions of hippocampus mainly focused on the pyramidal cell layer and the molecular layer. The protrusions of positive cells in striatum and hippocampus were long and the cell body 
was slightly larger, while the positive cells in the gyrus were small with large thin protrusion and distributed radially. The expression of GFAP-positive cells was slightly enhanced than before; the positive cells were large and stained deeply whose protrusions elongated and thickened. The expression of GFAP positive cells decreased at 24 hours in different brain region, especially the region of hippocampus. The expression of positive cells reached the lowest point at 3 days in the cortex and striatum, few small and slightly stained cells whose protrusions were few and thin were observed. The expression of GFAP positive cells at the same time point in hippocampus at $7 \sim 21$ days was stronger than the cortex ans striatum. It is approximate to each other at 28 days in different brain region. In the severe HI group (1I), GFAP positive cells were visible at 0.5 hours in different brain region. The morphological changes were close to the mild HI group at the same time point. The expression of positive cells was stronger than the mild $\mathrm{HI}$ group at $0.5 \sim 6$ hours and 7 28 days in different brain region. Also the decreasing degree was more prominent at 24 hours in hippocampus and at 3 days in cortex and striatum. GFAP-positive cells dropped to the lowest point at 3 days in the striatum, gradually increased at 7 28 days, the number of positive cells at each time point were significantly higher than the control group. The number of positive cells in the severe HI group were significantly higher than the mild HI group at 0.5 hours and 3 hours and was significantly higher than the control group and the mild HI group at postnatal 6hours, 7, 14, 21,28 days. The number of positive cells was significantly higher than the control group at 24 hours, but was not significantly different from those of the mild HI group. The number of positive cells was significantly lower than the mild HI group at 3 days, but was not significantly different from those of the control group.(Table 1).The expression of GFAP positive cells dropped to the lowest point at 3 days in the cortex and gradually increased 7 28 days. There is no difference between the control groups at 3 days, but the number of positive cells was significantly higher than the control group at other time points. The number of positive cells in the severe HI group were significantly higher than the mild HI group at 0.5 hours and 3 hours and was significantly higher than the control group and the mild HI group at postnatal 6 hours, 14, 21,28 days. The number of positive cells was significantly higher than the control group at 24 hours and 7 days, but was not significantly different from those of the mild HI group. The number of positive cells was significantly lower than the mild HI group at 3 days, but was not significantly different from those of the control group. (Table 2). GFAP positive cells dropped to the lowest point at 24 hours in the hippocampus and gradually increased after that. The number of positive cells was significantly higher than the control group at every time point. The number of positive cells in the severe HI group were significantly higher than the mild HI group at 0.5 hours and 3 hours and was significantly higher than the control group and the mild HI group at postnatal 6 hours, 7, 14, 21, 28 days. The number of positive cells was significantly lower than the mild HI group at 24 hours, but was not significantly different from those of the control group. The number of positive cells was significantly higher than the control group at 3 days, but was not significantly different from those of the mild HI group.(Table 3).

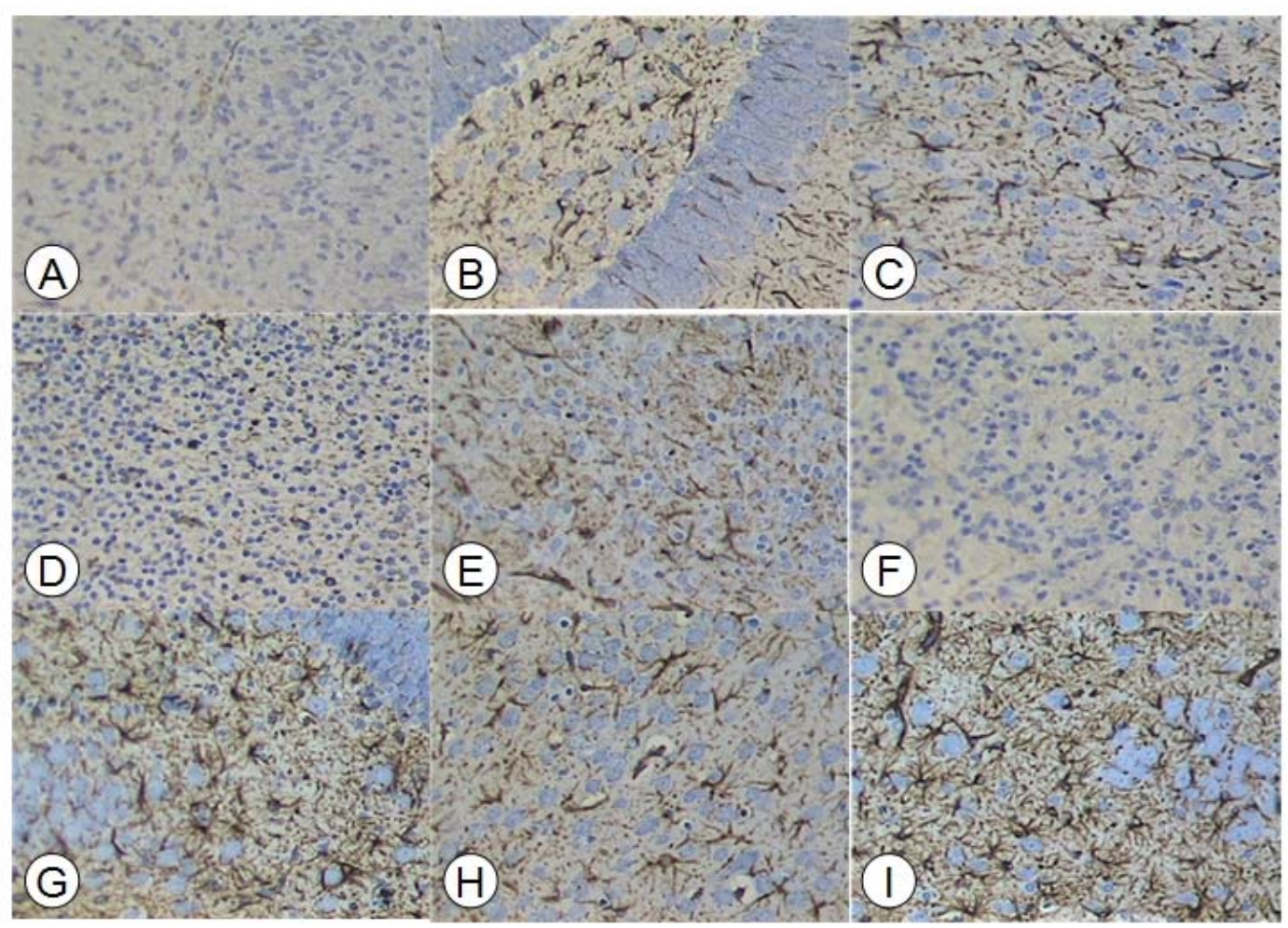

FIGURE I. IMMUNOHISTOCHEMICAL IMAGES OF CHANGES IN GLIAL FIBRIALLARY ACIDIC PROTEIN (GFAP)-POSITIVE CELL 
TABLE I. THE STRIATUM OVER TIME (0.5H-28D) IN NEONATAL WISTAR RATS FOLLOWING INTRAUTERINE ISCHEMIA

\begin{tabular}{|c|c|c|c|c|c|c|}
\hline Postnatal age & Number & Control group & Mild HI group & Severe HI group & P-valu & \\
\hline $0.5 \mathrm{~h}$ & 10 & 0 & $8.6 \pm 2.1^{\Delta}$ & $15.6 \pm 3.2^{\Delta} \boldsymbol{\Lambda}$ & 5.7833 & $<0.001$ \\
\hline $3 \mathrm{~h}$ & 10 & 0 & $12.5 \pm 2.7 \Delta$ & $20.1 \pm 3.7 \Delta \boldsymbol{\Delta}$ & 5.2470 & $<0.001$ \\
\hline $6 \mathrm{~h}$ & 10 & $3.7 \pm 0.6$ & $28.9 \pm 4.5 \Delta$ & $38.6 \pm 4.7 \Delta \boldsymbol{\Lambda}$ & 228.0 & $<0.001$ \\
\hline $24 \mathrm{~h}$ & 10 & $4.2 \pm 1.2$ & $18.4 \pm 3.9 \Delta$ & $20.1 \pm 4.1 \Delta$ & 68.34 & $<0.001$ \\
\hline $3 \mathrm{~d}$ & 10 & $4.6 \pm 1.5$ & $10.6 \pm 2.7 \Delta$ & $3.2 \pm 1.3 \mathbf{\Delta}$ & 41.2 & $<0.001$ \\
\hline $7 \mathrm{~d}$ & 10 & $5.6 \pm 1.3$ & $15.5 \pm 3.2 \Delta$ & $22.6 \pm 3.1 \Delta \boldsymbol{\Delta}$ & 101.54 & $<0.001$ \\
\hline $14 \mathrm{~d}$ & 10 & $7.6 \pm 2.4$ & $19.2 \pm 4.1 \Delta$ & $31.3 \pm 4.6 \triangle \boldsymbol{\Delta}$ & 96.35 & $<0.001$ \\
\hline $21 \mathrm{~d}$ & 10 & $20.2 \pm 3.7$ & $30.7 \pm 4.5 \Delta$ & $42.5 \pm 5.7 \Delta \boldsymbol{\Delta}$ & 59.89 & $<0.001$ \\
\hline $28 \mathrm{~d}$ & 10 & $26.5 \pm 3.9$ & $39.2 \pm 5.4 \Delta$ & $56.7 \pm 5.9 \Delta \boldsymbol{\Delta}$ & 87.12 & $<0.001$ \\
\hline
\end{tabular}

TABLE II. THE CORTEX OVER TIME (0.5H-28D) IN NEONATAL WISTAR RATS FOLLOWING INTRAUTERINE ISCHEMIA

\begin{tabular}{|c|c|c|c|c|c|c|}
\hline $\begin{array}{l}\text { Postnatal age } \\
\text { P-value }\end{array}$ & Number & Control & Mild $\mathrm{H}$ & Severe & HI group & $\mathrm{F}$ \\
\hline $0.5 \mathrm{~h}$ & 10 & $\overline{0}$ & $9.6 \pm 1.9^{\Delta}$ & $17.6 \pm 3.6^{\Delta}$ & 6.2148 & $<0.001$ \\
\hline $\begin{array}{l}3 h \\
6 h\end{array}$ & $\begin{array}{l}10 \\
10\end{array}$ & $\begin{array}{c}\mathbf{0} \\
4.7 \pm 1.1\end{array}$ & $\begin{array}{l}14.5 \pm 2.6^{\Delta} \\
23.9 \pm 4.1^{\Delta}\end{array}$ & $\begin{array}{l}26.1 \pm 3.9^{\Delta} \Delta \\
36.6 \pm 4.2^{\Delta} \Delta\end{array}$ & $\begin{array}{l}7.8261 \\
216.99\end{array}$ & $\begin{array}{l}<0.001 \\
<0.001\end{array}$ \\
\hline $24 h$ & 10 & $5.2 \pm 1.7$ & $19.4 \pm 3.6^{\Delta}$ & $18.1 \pm 4.5^{\Delta}$ & 51.21 & $<0.001$ \\
\hline 3d & 10 & $5.6 \pm 1.8$ & $6.6 \pm 2.4$ & $5.2 \pm 1.8$ & 1.27 & 0.2959 \\
\hline $7 d$ & 10 & $6.6 \pm 1.5$ & $14.5 \pm 2.2^{\Delta}$ & $16.6 \pm 2.1^{\Delta}$ & 72.53 & $<0.001$ \\
\hline 14d & 10 & $8.6 \pm 2.4$ & $18.2 \pm 4.6^{\Delta}$ & $29.3 \pm 4.3^{\Delta} \Delta$ & 70.89 & $<0.001$ \\
\hline 21d & 10 & $16.2 \pm 3.5$ & $25.7 \pm 3.7^{\Delta}$ & $37.5 \pm 4.7^{\Delta} \Delta$ & 71.2 & $<0.001$ \\
\hline $28 d$ & 10 & $20.5 \pm 3.8$ & $31.2 \pm 4.4^{\Delta}$ & $47.7 \pm 4.9^{\Delta \mathbf{\Lambda}}$ & 98.72 & $<0.001$ \\
\hline $\begin{array}{l}{ }^{*} \text { compared } \\
\mathrm{p}<0.001), \mathrm{HI}\end{array}$ & $\begin{array}{l}\text { control } \\
\text { xia-isch }\end{array}$ & $\begin{array}{ll}\text { roup } & \mathrm{p}<0.05 \\
\text { mia } & \end{array}$ & $5(? \mathrm{p}<0.001), \dagger \mathrm{cc}$ & ared with mild $\mathrm{HI}$ & I group & $\mathrm{p}<0.05$ \\
\hline
\end{tabular}

TABLE III. THE HIPPOCAMPUS OVER TIME (0.5H-28D) IN NEONATAL WISTAR RATS FOLLOWING INTRAUTERINE ISCHEMIA

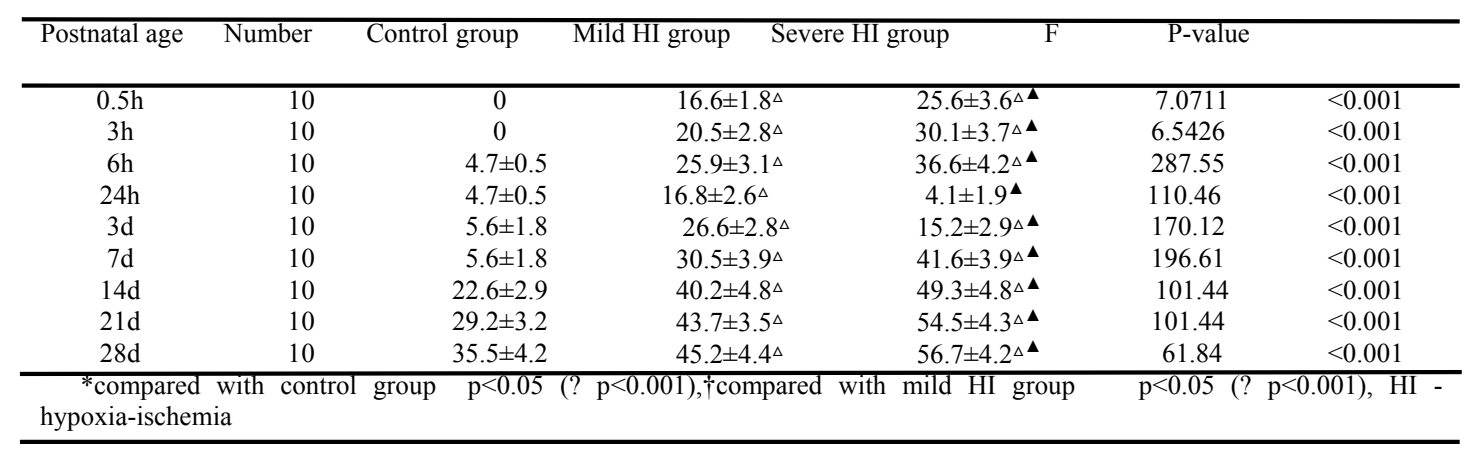

\section{DISCUSSION}

This study showed that GFAP positive astrocytes displayed differently among HI group the control group and the same time point between different HI groups. Compared to the control group at the same time point, the hyperplasia of GFAP positive astrocytes in the HI group was significantly higher and deeply stained. The GFAP positive astrocytes began to express at 0.5 hours in the HI group, and changes are as follows with the prolongation of reperfusion: their number increased, the cell body enlarged, the protrusions elongated and thickened and the staining deepened. However, not only the variation degree is different in different brain regions of the same HI group at the same time point, but the morphology is also inconsistent. The expression of GFAP positive astrocytes in the severe HI group was significantly decreased at 3 days of reperfusion in the striatum and cortex, while the region of hippocampus occurred above changes after 24 hours of reperfusion, after that GFAP positive astrocytes gradually increased in every region of the brain. The variation pattern is adapted to the functional status of astrocytes [3]. The changes of astrocytes after HIBD had two-way effect on neurons. Nevertheless, its effect of damaging or protecting neurons depends on the time and location of HI, which relies on the changes of the surrounding microenvironment and activating factors. Our study showed that GFAP positive astrocytes began to express lightly at postnatal 6 hours in the control group, whereas the GFAP positive cells began to appear at 0.5 hours in the HI group. The expression of GFAP positive astrocytes in the striatum and cortex gradually increased at $0.5 \sim 24$ hours and reached the lowest point at 3 days, but the region of the hippocampus reached the lowest point at 24 hours, after that all 
regions of the brain were gradually increased. In our study, ischemia and hypoxia made GFAP positive astrocytes reactive hyperplasia earlier that is relevant to protective effect of astrocytes on neuron. Astrocytes can release large amounts of neurotrophic factors and neurotransmitters which plays an important role in the repair of nerve injury[4]. The cell membrane broken since glutamate, nitric oxide, oxygen free radicals continued to accumulate in the astrocytes and the influence of hypoglycemia, acidosis as a result of cell death. Therefor it can explain the expression of GFAP positive astrocytes after reperfusion 3 days in the striatum and cortex and after reperfusion 24 hours in hippocampus significantly reduced. The GFAP positive astrocytes decreased with more severity in severe HI group. As further release of $\mathrm{K}+$, glutamic acid, lactic acid, free radicals and arachidonic acid and other substances by the injury brain tissue can also lead to new astrocytes hyperplasia[6]. Due to the damaged neurons in this period has been significantly reduced compared with the previous, so the astrocytes performed sustained hyperplasia. Astrocytes can release large amounts of neurotrophic factors and cytokines, so it plays an important role in the repair of the nerve injury $[6,7]$.

In short, early stage of different degrees of HI can promote the proliferation of astrocytes and developed the neuroprotective effect. But overactive astrocytes will lead to glutamic acid, nitric oxide, oxygen radicals increasing continually and the release of abundant harmful factors, which not only brought about the death of themselves, meanwhile also had adverse effect on other neural cells.

\section{REFERENCE}

[1] Bo T, Yan C, Huo S, Li W. Reconstructing new animal model of peri-delivery hypoxia ischemia brain damage by ligaturing bilateral uterine arteries of pregnant mouse. Journal of Apoplexy and Nervous Diseases 2001; 18: 38-39. Chinese.

[2] Wu H,Wang DX,Wang GH,et al.Temporal and spatial changes in oligodendrocytes in different brain regions following intrauterine ischemia in neonatal Wistar rats2013;18:447.

[3] Ouyang YB, Voloboueva LA, Xu LJ, et al. Selective dysfunction of hippocampal CA1 astrocytes contributes to delayed neuronal damage after transient forebrain ischemia. J Neurosci. 2007 Apr 18; 27(16):4253-4260.

[4] Sofroniew MV. Reactive astrocytes in neural repair and protection [J].neuroscientist, 2005, 11(5):400-407.

[5] Kovalenko TM, Osadchenko IO, Tsupykov OM, et al. Neuroprotective effect of quercetin during experimental brain ischemia. Fiziol Zh, 2006, 52(5): 21-27.

[6] Xu A, Wang Y, Xu LY, et al. Protein Kinase Calpha mediated negative feedback regulation is responsible for the termination of insulin like growth factor-I induced activation of nuclear phospholipase $\mathrm{C}$ betxl in swiss 3T3 cells. Biochem, 2001, 276(18):14980-14986.

[7] Lau LT, Yu AC. Astrocytes produce and release interleukin-1, interleukin-6, tumor necrosis factor alpha and interferon-gamma following traumatic and metabolic injury. J Neurotrauma, 2001, 18(3): 351-359. 